\title{
Cybersecurity Requirements for AM Systems
}

\author{
New Enforcement in DoD Environments, and Resources for Implementation
}

\author{
Mark J. Cotteleer \\ Deloitte Consulting LLP \\ Milwaukee WI USA \\ mcotteleer@deloitte.com \\ Oyindamola Alliyu \\ Deloitte Consulting LLP \\ Arlington VA USA \\ oalliyu@deloitte.com
}

\author{
Simon S. Goldenberg ${ }^{\dagger}$ \\ Deloitte \& Touche LLP \\ Arlington VA USA \\ sigoldenberg@deloitte.com
Stephen Kania
Deloitte Consulting LLP
Arlington VA USA
skania@deloitte.com \\ Brenna Sniderman \\ Deloitte Services LP \\ Philadelphia PA USA \\ bsniderman@deloitte.com
}

\author{
Ian Wing \\ Deloitte Consulting LLP \\ Arlington VA USA \\ iwing@deloitte.com \\ Veda Mujumdar \\ Deloitte \& Touche LLP \\ Arlington VA USA \\ vmujumdar@deloitte.com
}

\begin{abstract}
The Office of the Inspector General (OIG) for the US Department of Defense (DoD) released Audit of the Cybersecurity of Department of Defense Additive Manufacturing Systems (DODIG-2021-098) [1] in July 2021, to determine "whether DoD [sites] secured additive manufacturing (AM) systems to prevent unauthorized changes and ensure the integrity of the design data." The audit report recommends requiring "all AM systems to obtain an authority to operate in accordance with DoD policy before their use" [1], and requiring "AM system owners to immediately identify and implement security controls to minimize risk until obtaining an authority to operate." [1] The DoD Chief Information Officer (CIO) responded that existing DoD regulations require both of these for "all IT systems, including AM systems" [1]. Such cyber security rules can help guard against vulnerabilities such as design file theft or digital thread hacking, as well as unauthorized prints on AM systems that can impact the safety and integrity of parts used in defense systems, expose critical intellectual property to bad actors and even cause manufacturing facilities to shut down. To improve AM system vendors' understanding of these cybersecurity requirements for DoD and the US Government (USG), we discuss in this paper the process for obtaining an Authority To Operate (ATO) certification for an IT system per DoD and USG cybersecurity regulations, i.e., the Risk Management Framework (RMF) process from the US National Institute of Standards and Technology (NIST) [2]. We also describe resources for AM system vendors to understand and implement the RMF

\section{${ }^{\dagger}$ Correspondence Author}

Permission to make digital or hard copies of part or all of this work for personal or classroom use is granted without fee provided that copies are not made or distributed for profit or commercial advantage and that copies bear this notice and the full citation on the first page. Copyrights for third-party components of this work must be honored. For all other uses, contact the Owner/Author(s). AMSec '21, November 19, 2021, Virtual Event, Republic of Korea. (c) 2021 Copyright is held by the owner/author(s). ACM ISBN 978-1-4503-8480-3/21/11. https://doi.org/10.1145/3462223.3485624
\end{abstract}

process for obtaining an ATO certification, particularly in the DoD environment.

\section{CCS CONCEPTS}

- Security and Privacy - Formal methods and theory of security - Security Requirements

\section{KEYWORDS}

AM security; additive manufacturing; 3D printing; security; Risk Management Framework; NIST RMF; Department of Defense; DoD

ACM Reference Format:

Mark J. Cotteleer, Simon S. Goldenberg, Ian Wing, Oyindamola Alliyu, Stephen Kania, Veda Mujumdar, \& Brenna Sniderman. 2021. Cybersecurity Requirements for AM Systems: New Enforcement in DoD Environments, and Resources for Implementation. In Proceedings of the 2021 Workshop on Additive Manufacturing (3D Printing) Security (AMSec '21), November 19, 2021, Virtual Event, Republic of Korea. ACM, New York, NY, USA, 12 pages. https://doi.org/10.1145/3462223.3485624

\section{Introduction: AM and Cybersecurity}

Additive Manufacturing (AM), commonly known as 3D-printing, has an urgent need for cybersecurity. AM allows parts to be printed where they are needed, replacing physical delivery of material with digital instructions that can be transmitted to machines co-located closest to assembly or to the assets requiring repairs. While the 3D printing process has developed and improved to enable more widespread use, concerns around security have also broadened beyond hackers stealing designs to exploit data and information, or to create counterfeit products. New concerns continue to surface, such as bad actors seeking to inflict harm or damage to an operation through tampering [3], or through the injection of undetectable and potentially devasting flaws in products [4]. For agencies and organizations deploying 
AM technology, acknowledging this reality is not just a business or operational imperative, but potentially a matter of life and death, particularly for medical, defense, or national security applications.

\subsection{Cyberattacks are Proliferating}

Cyberattacks are on the rise, and their costs also continue to climb. The National Vulnerability Database (NVD) reports that more than 17,000 computer vulnerabilities were identified in 2019, and more than 18,000 in 2020 [5]. The Center for Strategic and International Studies (CSIS) published a report on "The Hidden Costs of Cybercrime" in 2020 [6] which estimated that the global losses from cybercrime approach $\$ 1$ trillion, up from the $\$ 600$ billion CSIS reported for 2017. Cybersecurity vulnerabilities extend beyond just traditional computers, and can impact logic bearing and data storing devices in general. A recent cyber-attack on an industrial control system in a municipal water system [7], which nearly caused changes to water plant output, illustrates the potential exposure of AM and Industry 4.0 systems. In response to the widely-reported ransomware attack against an oil pipeline in the US earlier this year [8], the owners “... proactively took certain systems offline to contain the threat, which temporarily halted all pipeline operations, and affected some of our IT systems" [8], demonstrating effects on operational systems of attacks on related IT systems. Appropriate cybersecurity measures will be necessary to abate the damages that could result from possible attacks. An effective response to this volume of attacks, and to profitably motivated cyber criminals, requires a systematic and organized approach.

\subsection{Vulnerabilities of AM systems}

$\mathrm{AM}$ is a set of industrial production technologies that can turn digital designs into physical products. AM can not only provide flexibility of manufacture, in both location (e.g., near the point of use), and time (e.g., on demand) [9], but can also allow for more structurally complex builds, in terms of design and material use, than subtractive manufacturing systems can produce. The number of 3D-printed components grows each day. These parts make their way into vehicles, industrial machinery, medical devices, and military applications. With that increase comes more opportunities for malicious hacks into AM industry, potentially jeopardizing the products, and consequently potentially impacting safety, health, or mission objectives. Figure 1 [9][10][11] depicts the AM process characterized by a digital thread which covers the design, build, quality assurance testing, final delivery, and distribution of 3D printed parts. Within each step of this process, there are vulnerabilities, cyber risks, or digital threats related to intellectual property, software, network, printers, production and more. The connectivity-intensive nature of the full AM process and associated technologies makes it nearly impossible to avoid such threats.

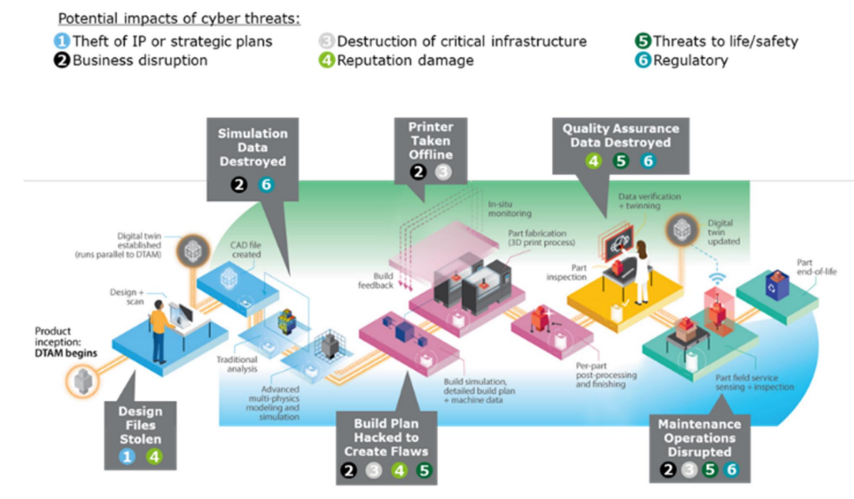

Figure 1: AM Vulnerabilities illustrated via the Digital Thread [9][10][11]

\section{DoD IG Cybersecurity Audit of AM Systems}

In recent years, AM has become an integral part of the DoD's mission readiness [12]. DoD uses AM for production of parts within a range of areas from weapon systems and arms to body implants and prosthetics. Although AM is a valuable manufacturing tool for the DoD, the potential for cybersecurity threats to AM systems (such as shown in Figure 1) has caused increasing concern for the DoD.

As part of its audit (from April 2019 through March 2021), the Office of the Inspector General (OIG) visited five DoD sites to inspect the cybersecurity controls of AM systems, defined as printers and computer workstations used to develop and print three-dimensional products [1]. In total, OIG reviewed $73 \mathrm{AM}$ printers and 46 computers, three of which were computers built into AM printers [1]. To determine the baseline security controls appropriate for protecting information systems, the DoD IG used National Institute of Standards and Technology (NIST) Special Publication (SP) 800-53, Revision 4 [13], and NIST Internal Report (NISTIR) 8183 [14]. The OIG review focused on seven cybersecurity criteria, the absence of which OIG determined present a higher-risk to AM systems and DoD: Operating System Updates; Use of Authentication Factors; Unauthorized User Accounts; Vulnerability Identification; Protection of Removable Media; Property Accountability; and Implementation of Physical Security [1].

Upon this review of the cybersecurity measures of DoD AM systems, the IG found that DoD officials at the audited sites "did not consistently secure or manage their AM systems to prevent unauthorized changes and ensure the integrity of the design data" [1], and consequently that "DoD [sites] were unaware of existing AM system vulnerabilities that exposed the DoD Information Network (DODIN) to unnecessary cybersecurity risks." [1] Such risks include, but are not limited to, malicious actors compromising AM systems by introducing internal defects to the manufacturing process; stealing or altering design data, potentially harming the integrity of printed parts; and gaining unauthorized access to DoD information networks [1]. The IG also noted that data compromises could allow adversaries to reverse-engineer and use stolen DoD parts to their advantage on the battlefield, or to 
change AM design data, potentially affecting the strength and utility of AM products [1].

Based on the OIG audit findings, the IG audit report makes the following recommendations for improving the cybersecurity of DoD's AM systems:

1. That additive manufacturing systems be included in the DoD information technology systems portfolio and establish and maintain cybersecurity controls in accordance with Federal and DoD guidance. [1]

2. That the DoD Chief Information Officer (CIO) require additive manufacturing system owners to immediately identify and implement security controls to minimize risk until obtaining an authority to operate. [1]

3. That all additive manufacturing systems be required to obtain an authority to operate in accordance with DoD policy before their use. [1]

The DoD CIO's response to these recommendations was that all three of them were already required for AM systems, since they are IT systems. [1]

Therefore, AM system vendors to DoD must be aware of RMF cybersecurity requirements and comply with them. As this is an unfamiliar process for many AM vendors, the following sections provide information about the ATO process, with additional notes for AM systems for DoD.

\section{RMF Process}

In this section, we outline the NIST RMF process [15] for obtaining an ATO certification for an IT system deployed on behalf of the US Government (USG), focusing on DoD deployments, and elaborating on how these steps and procedures apply to AM systems.

The legal basis for the RMF is the Federal Information Security Modernization Act of 2014 (FISMA) [16]. FISMA directed NIST to develop the cybersecurity standards that became the RMF, and FISMA mandates USG IT systems comply with security standards that NIST develops. Per FIMSA, NIST developed Federal Information Processing Standard (FIPS) 200 "Minimum Security Requirements for Federal Information and Information Systems" [17], which requires compliance with the RMF for USG IT systems. There are exceptions for some national security systems, which have their own standards [17] [18]. DoD updated their previous cybersecurity requirements framework to comply with the RMF in DoD Instructions 8500.01 [19] and 8510.01 [20].

It is worth reviewing the USG definition of IT, and therefore an IT system, since USG [17] and DoD [19] regulations require the RMF for systems that meet this definition. The Committee on National Security Systems Instruction (CNNSI) 4009, "National Information Assurance Glossary" [21] definition of information technology is in Figure 2.

This definition does not specify that the IT system reside in a government environment, if the IT system processes "data or information by the executive agency" [21]. Note that this definition is broad enough to include traditional IT, AM systems,
Any equipment or interconnected system or subsystem of equipment that is used in the automatic acquisition, storage, manipulation, management, movement, control, display, switching, interchange, transmission, or reception of data or information by the executive agency. The term information technology includes computers, ancillary equipment, software, firmware and similar procedures, services (including support services), and related resources.

Figure 2: USG Definition of Information Technology [21]

and Internet of Things devices. Additionally, RMF compliance may be specified in USG contracts.

\subsection{Cybersecurity Objectives of the RMF}

US law [22] defines information security as "protecting information and information systems from unauthorized access, use, disclosure, disruption, modification, or destruction in order to provide" the three objectives of Confidentiality, Integrity, and Availability. These objectives are often referred to as the "CIA Triad". The definition of these objectives from [22] are shown in Figure 3:

\begin{tabular}{|l|l|}
\hline Confidentiality & $\begin{array}{l}\text { "preserving authorized restrictions on access } \\
\text { and disclosure, including means for protecting } \\
\text { personal privacy and proprietary information" }\end{array}$ \\
\hline Integrity & $\begin{array}{l}\text { "guarding against improper information } \\
\text { modification or destruction, and includes } \\
\text { ensuring information nonrepudiation and } \\
\text { authenticity" }\end{array}$ \\
\hline Availability & $\begin{array}{l}\text { "ensuring timely and reliable access to and use } \\
\text { of information" }\end{array}$ \\
\hline
\end{tabular}

Figure 3: CIA Triad Definitions [22]

\subsection{RMF Process Steps}

The RMF process is defined in NIST SP 800-37, "Guide for Applying the Risk Management Framework to Federal Information Systems" [15]. Although the RMF is a USG standard, and mandated for systems deployed on behalf of USG agencies, the RMF is an openly published standard, and may be adopted by any organization. There is flexibility in how the guidance is implemented, allowing agencies to tailor their approaches according to their mission, business processes, and environment. Thus, the RMF implementation can differ from agency to agency. However, the underlying objective stays the same: to help organization's processes and systems to become resilient and recover quickly from security incidents.

SP-800-37 provides a comprehensive, flexible, repeatable, and measurable 7-step process that any organization can use to manage information security and privacy risk for organizations and information systems. Overall, the RMF process can take 6-12 months to obtain an ATO certification. The 7-step process is shown in Figure 4 below: 


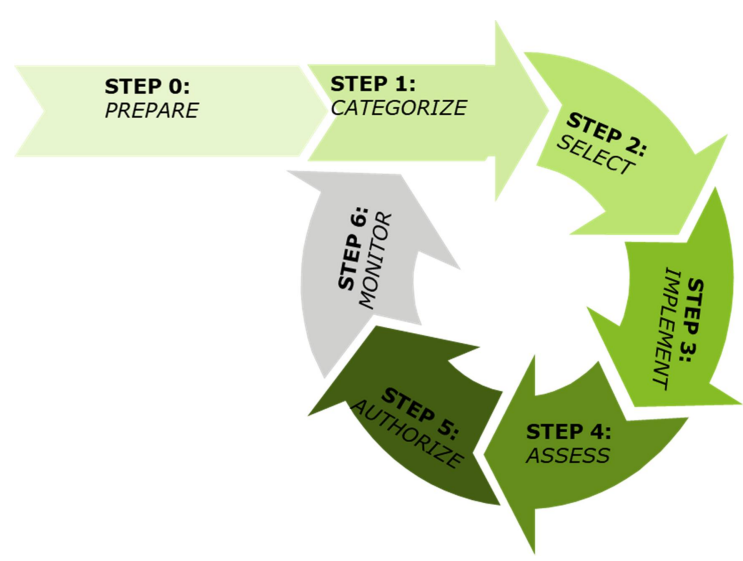

Figure 4: NIST RMF 7-Step Process [15] [23]

NIST provides more information about each of the RMF Steps on their web site [15]. Briefly summarizing the RMF Steps:

1. Step 0 - Prepare: Identify roles, business function, system accreditation boundary, and other system characteristics. [15]

2. Step 1 - Categorize: Establish Low, Moderate, or High categorization of system based on information type. [15]

3. Step 2 - Select: Establish security control list based on categorization and system characteristics. [15]

4. Step 3 -: Implement: Implement security controls in system and develop required security documentation. [15]

5. Step 4 - Assess: Conduct assessments and remediate findings. Generate mitigation plans as necessary. [15]

6. Step 5 - Authorize: Review ATO package and provide authorization decision. [15]

7. Step 6 - Monitor: Continue on-going authorization and manual assessments. Provide security status reports to designated roles. [15]

The RMF process is designed to be technology-neutral, and there is very little about this overview of the process that would be different for AM systems, versus any other IT system. The differences for AM systems would be in the details of the selected controls, which is discussed in Section 4, and in the approach of the control assessment, which is outside the scope of this paper.

We will discuss the Categorize step (Step 1), and the Monitor step (Step 6) in Sections 3.3 and 3.5 respectively. In Section 3.4 we discuss Mitigations and Risk Acceptances, which impact RMF Steps 3, 4, and 5. We finish the topic of the RMF Process with a discussion of RMF Roles in Section 3.6.

\subsection{RMF Security Categorizations}

Different federal agencies handle the Categorize Step (Step 1) [15] differently, depending upon their mission and business functions, contractual and legal obligations, and other considerations. According to Federal Information Processing Standard (FIPS) 199 "Standards for Security Categorization of Federal Information and Information Systems" [24], security categorization standards for information and information systems "provide a common framework and understanding for expressing security that, for the federal government, promotes:
1. Effective management and oversight of information security programs, including the coordination of information security efforts throughout the civilian, national security, emergency preparedness, homeland security, and law enforcement communities; and

2. Consistent reporting to the Office of Management and Budget (OMB) and Congress on the adequacy and effectiveness of information security policies, procedures, and practices." [24]

FIPS 199 [24] describes a security categorization approach in which the system is evaluated for the potential "adverse effect on organizational operations, organizational assets, or individuals" an unauthorized disclosure of information from the system would have on each of the three Security Objectives: Confidentiality, Integrity, and Availability. Potential adverse effects that are "limited" are categorized as Low, "serious" effects are categorized as "Moderate", and "severe or catastrophic" are categorized as "High" [24]. The categorization for each security objective is done based on the information types in the system [24]. Both NIST SP 800-60 [25] and FIPS 200 [17] extend the procedures in FIPS 199 by providing guidance for consolidating the three-part impact level to a single value (the highest rating among the three security objectives) and using that overall system security categorization as the basis for the Select Step (Step 2) [15] by selecting a security control baseline to satisfy minimum security requirements. This high watermark approach is commonly used by civilian USG agencies who have few National Security Systems (NSS).

Another approach is described in Committee on National Security Systems Instruction No. 1253 (CNSSI 1253) "Security Categorization and Control Selection for National Security Systems" [18]. CNSSI 1253 provides guidance for security categorization of NSS that collect, generate, process, store, display, transmit, or receive National Security Information [18]. CNSSI 1253 adopts a similar approach as FIPS 199 for the information type impact levels, but does not consolidate system-level confidentiality, integrity, and availability ratings to a single impact designation [18]. Instead, NSS categorized using CNSSI 1253 maintain their three-part categorization ratings for the security control selection process [18]. CNSSI 1253 also adapts the security control baselines in NIST SP 800-53 to indicate control applicability at each impact level and each security objective [18]. This three-part categorization approach is used by DoD, by USG agencies with national security missions, and by USG agencies for their NSS.

Within these two approaches, USG or DoD agencies may have criteria for categorizing systems based of system data, or other characteristics including supply chain consideration, and may specify the system categorization in the contract for purchasing the system.

\subsection{Mitigations and Risk Acceptances}

An important, but less well-known, aspect of the RMF process is that a system does not have to be assessed as $100 \%$ compliant with the selected controls to obtain an ATO certification. During the Implement (Step 3) and Assess (Step 4) steps, controls for which the system is not in compliance can be addressed in two ways. 
Risk Acceptance Requests (RARs) are designed for controls that cannot be fixed due to technology limits, or for which the fixes will be too expensive as decided by the Authorizing Official (AO) [15]. Controls that can be made compliant, but not in time for the ATO date, must be put under a mitigation plan, called a Plan of Actions and Milestones (POA\&M) [15]. The POA\&Ms and RARs are included in a system's ATO authorization package at the end of the Assess Step (Step 4), along with the assessment results and system documentation, for $\mathrm{AO}$ review and approval in the Authorize Step (Step 5) [15]. The AO has the authority to reject POA\&Ms or RARs [15], so it is important for AM vendors to work closely with their DoD cybersecurity counterparts to make sure the POA\&Ms and RARs are acceptable to the AO for the client agency.

\subsection{Cybersecurity Monitoring in the RMF}

The Monitor Step (Step 6) [15] acknowledges that security is not an endpoint, but an ongoing process. New threats constantly arise and need to be addressed. Here, stakeholders focus on regular review of the system's security posture. Traditionally, an ATO (issued in RMF Step 5 by the AO) is valid for no more than three years and includes security re-assessments of some of the controls one year and two years after the ATO was issued, in line with annual reporting requirements [26]. The system then undergoes an entire re-assessment in the third year. A newer approach, called Continuous Monitoring or Continuous Authorization, frequently (e.g., monthly) reassesses a sub-set of the selected controls for the system [26]. As long as the system's security posture remains satisfactory, the ATO can be extended on an ongoing basis [26]. AM vendors should ascertain which Monitor Step approach their client agency is using.

\subsection{RMF Roles}

Each RMF Step depicted in Figure 4 corresponds to a relevant set of personnel in the DoD or USG agency. Depending upon the organization size, internal structure, and program offices alignment, these roles can differ and overlap in the ways in which they support RMF steps. SP 800-37 [15] has more details on the roles and responsibilities for each step. Some of these roles are explicitly supporting the system vendor, whether the system is traditional IT, AM, or Industry 4.0. For example, the System Owners/Information System Owners (SOs/ISOs) and Information System Security Officers (ISSOs) roles are designed to support the vendor in the RMF process. Although the Security Control Assessment Team is designed to be independent of both the vendor and the vendor's DoD/USG contacts (SOs/ISOs, ISSOs, and Contracting Officer), the assessment team can sometimes be a resource for advice on control compliance. Government personnel with RMF experience can be effective guides to the specific agency's RMF process, particularly for vendors without RMF experience, or RMF experience at that agency. Given that the DoD IG's report requiring ATO certification for AM systems was released only a few months ago [1], AM vendors would not necessarily have RMF experience.

\section{Cybersecurity Controls in the RMF}

Appreciation of the full scope of the cybersecurity requirements imposed by the NIST RMF necessitates not only an understanding of the RMF process outlined in the prior section, but also of the specific cybersecurity controls required by the RMF. The catalog of cybersecurity controls for the RMF is contained in SP 800-53 [13]. Revision 4 of this catalog is the version currently in use within DoD. RMF Privacy controls will be discussed in Section 4.5.

SP 800-53 defines security controls as "the safeguards / countermeasures prescribed for information systems or organizations that are designed to: (i) protect the confidentiality, integrity, and availability of information that is processed, stored, and transmitted by those systems/organizations; and (ii) satisfy a set of defined security requirements." [13] NIST has designed the RMF security controls to be technology-neutral and policy-neutral, so that the controls "focus on the fundamental safeguards and countermeasures necessary to protect information during processing, while in storage, and during transmission." [13] This technology and policy-neutral approach has led to most of the RMF security controls being high-level and general, rather than overly specific, as will be seen in the examples in Section 4.4, below. RMF's technology and policy neutrality must have been one of the factors that led the DoD CIO to determine that specific guidance did not have to be established for the RMF to apply to AM systems.

\subsection{RMF Control Responsibility}

RMF Security controls for which the IT system, or the personnel supporting or managing the system, are solely responsible for the implementation of are called system-specific controls [13]. For example, a system's administrators may be solely responsible for installing patches and upgrades.

Controls for which another system or organization is responsible for the implementation, even when the control is selected for the system, are called common controls [13]. The system inherits the implementation of the control from the other system, which is called a common control provider [13]. If, for example, an AM system is installed in a government office or data center, then the AM system would not be held responsible for the implementation of common controls such as physical security and environmental protection (temperature, humidity, power), or network security. The government would be responsible for those controls, and the AM system would inherit their implementation. However, if an AM system is installed in a commercial facility, those controls would be considered as system-specific, and compliance would be the responsibility of the AM system, even if the facility is managed by a third party.

Security controls where some of the requirements are the responsibility of the system, and others are the responsibility of a common control provider are called hybrid controls [13]. An example of a hybrid control would be when end user access is managed by an enterprise-wide identity and access system (the common control provider), but managers for the system must designate which individuals should have their profiles updated to allow access to the system. 
Common control providers can be an effective means for reducing the number of controls a system is responsible for, which can significantly decrease the AM vendor's level of effort for obtaining an ATO certification. Common control providers often include network services, physical and environmental controls, security and privacy awareness and training, security assessment and authorization, planning, program management, personnel security, and risk assessment. Every control that is the responsibility of another system is one less control that the AM vendor must address, possibly with system or process changes. IT vendors, including AM vendors, may also develop their own common control providers, within their system. For example, a multi-server system that has a single patch management process for all servers may request that the patch management process for the system be made a "local" common control provider, and therefore related controls would have to be assessed just once in the ATO effort, rather than for each server in the system.

\subsection{RMF Security Control Families}

RMF controls are grouped into eighteen families, collecting security controls related to the general security topic of the family [13][17]. To introduce the reader to the security topics in the RMF, Figure 5 shows a list of the RMF Security control family names, and their abbreviations [13]. The control families that are more likely to apply to AM systems are indicated with a background color and bold typeface, although specific controls from other families may be apply to a specific AM system, or be required by a DoD agency, or may have shared responsibility between DoD and the AM vendor (hybrid controls).

\begin{tabular}{|l|}
\hline RMF Security Control Family Names and Abbreviations \\
NIST SP 800-53 Revision 4 [13] \\
\hline Access Control (AC) \\
\hline Awareness and Training (AT) \\
\hline Audit and Accountability (AU) \\
\hline Security Assessment and Authorization (CA) \\
\hline Configuration Management (CM) \\
\hline Contingency Planning (CP) \\
\hline Identification and Authentication (IA) \\
\hline Incident Response (IR) \\
\hline Maintenance (MA) \\
\hline Media Protection (MP) \\
\hline Physical and Environmental Protection (PE) \\
\hline Planning (PL) \\
\hline Program Management (PM) \\
\hline Personnel Security (PS) \\
\hline Risk Assessment (RA) \\
\hline System and Services Acquisition (SA) \\
\hline System and Communications Protection (SC) \\
\hline System and Information Integrity (SI) \\
\hline
\end{tabular}

Figure 5: RMF Security Control Family Names and Abbreviations [13]
We provide more detail in the following sections for some of the Security control families in the RMF. To focus on AM systems, this discussion of RMF control families is limited to those related to the IG audit report [1], or to examples discussed in Section 4.4. For each Security control family below, we provide a brief description of the topic area for each security control family, and a comment on the control family's impact on AM systems. The descriptions were adapted from FIPS 200 [17], which has more details, as does SP 800-53 [13].

\subsubsection{Access Control (AC) Control Family}

Security controls in the Access Control (AC) family limit information system access to authorized users, processes acting on behalf of authorized users, or devices (including other information systems) and to the types of transactions and functions that authorized users are permitted to exercise. [17]. These controls may be selected for AM systems to assess the security of access by human users, or by automated processes or systems. User authentication factors was one of the criteria the DoD IG audit used to determine AM system security [1].

\subsubsection{Audit and Accountability (AU) Control Family}

Security controls in the Audit and Accountability (AU) family ensure that the actions of individual information system users can be uniquely traced to those users so they can be held accountable for their actions, and to ensure the creation, protection, and retention of information system audit records needed to enable the monitoring, analysis, investigation, and reporting of unlawful, unauthorized, or inappropriate information system activity. [17]. System activity records can be important for investigating cyberattacks, attempted attacks, and anomalies. At least some AU family controls are usually selected for most systems, and AM systems would not be excepted.

\subsubsection{Security Assessment and Authorization (CA) Control Family}

Security controls in the Security Assessment and Authorization (CA) family ensure the USG agency is properly implementing the RMF process, and other closely related activities [17]. One of the CA controls is an example in Section 4.4.4, concerning data interconnections. The DoD IG noted in the audit report that AM systems often had data interconnections, which qualified them as IT systems subject to RMF requirements [1].

\subsubsection{Configuration Management (CM) Control Family}

Security controls in the Configuration Management (CM) family ensure establishment and maintenance of baseline configurations and inventories of information systems throughout the system development life cycle, and establishment and enforcement of security configuration settings in organizational information systems [17]. Maintaining configuration, and system inventory of AM systems, were among the concerns in the DoD IG audit report [1].

\subsubsection{Media Protection (MP) Control Family}

Security controls in the Media Protection (MP) family ensure protection of information system media, both paper and digital, limitation of access to information on information system media to authorized users, and sanitization or destruction of information 
system media before disposal or release for reuse [17]. Protection of removeable media for AM system was one of the criteria the DoD IG audit used to determine AM system security [1].

4.2.6 Physical and Environmental Protection (PE) Control Family

Security controls in the Physical and Environmental Protection (PE) family ensure limitation of physical access to information systems, equipment, and the respective operating environments to authorized individuals, protection of the physical plant and support infrastructure for information systems, provision of supporting utilities for information systems, protection of information systems against environmental hazards, and appropriate environmental controls in facilities containing information systems [17]. Physical and environmental protection of AM system was one of the criteria the DoD IG audit used to determine AM system security [1].

4.2.7 System and Information Integrity (SI) Control Family Security controls in the System and Information Integrity (SI) family ensure the identification, reporting, and correction of information and information system flaws in a timely manner, the protection from malicious code at appropriate locations within information systems, and monitoring information system security alerts and advisories and take appropriate actions in response [17]. The control family includes controls for flaw remediation, patching, and vulnerability scanning. Timely application of operating system updates for AM systems was one of the criteria the DoD IG audit used to determine AM system security [1].

\subsection{Documentation Controls}

The first control in each RMF Security control family concerns the development of policy documents for the topic of the control family [13]. While these controls apply to systems at any security categorization level [13], not all of them will be selected for a given system's RMF assessment. If a particular one of these controls was not selected for a system, the expectation is that the required policy document has previously been developed for the agency.

The eighteen policy documents required for agencies by these RMF controls are not the only policy documents required by the RMF. Additional policy documents are required by other controls which may be selected for a given system. A common RMF approach is to have high-level policy documents, more specific procedure documents, and in some cases checklists or implementation documents. Templates for these documents are often available from the USG agency, or from NIST, and the policy documents are typically short and straightforward. Another source of RMF documentation and templates is the Federal Risk and Authorization Management Program (FedRAMP). FedRAMP is a program for security authorizations for Cloud-based services under the RMF [27]. Although their focus is on Cloud-based services, FedRAMP publishes RMF documents and templates [28] that can be adapted for use in RMF efforts for on-premise systems such AM devices.

The proportion of documentation and process controls in the RMF Control catalog, over detailed technical controls, is a consequence of the RMF's technology-neutrality. The prevalence of policy and documentation controls in the RMF means there are RMF security controls that can be addressed with documentation or process changes, versus technical solutions. While technical solutions are more amenable to automation, which makes checking control compliance a repeatable process with reduced likelihood of human error, there are RMF controls that are solely documentation controls, and there are cases where a document or policy change can address a control's compliance in less time that it would take to develop, test, debug, and deploy a technical solution.

\subsection{RMF Control Examples for AM Systems}

Specific examples of RMF Security controls that may apply to AM systems deployed for DoD and USG make this discussion more concrete and support a more detailed understanding of the RMF. We first describe the numbering scheme used in the RMF for controls [13] in Section 4.4.1, and the information the RMF control catalog has for each control [13] in Section 4.4.2, before giving detailed examples in Sections 4.4.3 through 4.4.6. This information in Sections 4.4.1 and 4.4.2 supports an independent review of the RMF control catalog [13] by the reader.

\subsubsection{RMF Security Control Numbering Scheme}

Each RMF control is identified by a two letter Control Family abbreviation, a dash, and a Control Number up to 2 digits [13]. No control family has more than 99 controls. Control Numbers less than 10 do not have leading zeros in the NIST publications, e.g., AC-9 rather than AC-09 [13]. Some NIST Controls include Control Enhancements (additional checks for use on higher risk systems or by agency policy). Control enhancements are identified by the enhancement number in parentheses after the Control Number, e.g., AC-2(4) [13].

\subsubsection{RMF Control Contents}

The SP 800-53 control catalog lists, for each control [13]:

1. Control number and title,

2. Control text,

3. Supplemental guidance for the control,

4. Related controls,

5. Control enhancements, if any,

6. External references, if any,

7. NIST Priority rating for the control

8. NIST guidance on the applicability of the control and its enhancements to systems with Low, Moderate or High categorization.

Note that DoD systems and national security systems follow the guidance in CNSSI 1253 [18], and that USG agencies (including DoD agencies) are allowed under the RMF to change the applicable categorizations of RMF controls, as well as to add their own controls for their systems [13] [15]. Also note that the priority ratings have not been commonly used for RMF assessments in recent years.

4.4.3 Security Control AU-3 "Content of Audit Records" Our first example of an RMF security control is the third control in the Audit and Accountability family, designated AU-3 and titled "Content of Audit Records" [13]. This is a control that is frequently 
selected to apply to IT systems, and AM systems are unlikely to be excepted.

The control text for AU-3 is in Figure 6: [13]

The information system generates audit records containing information that establishes what type of event occurred, when the event occurred, where the event occurred, the source of the event, the outcome of the event, and the identity of any individuals or subjects associated with the event.

\section{Figure 6: Text of RMF control AU-3 [13]}

Auditing in the context of cybersecurity and the RMF is related to IT system activity, not only financial transactions. SP 800-53 includes the definition of an Audit Log as "A chronological record of information system activities, including records of system accesses and operations performed in a given period," [13] and an audit record as "An individual entry in an audit log related to an audited event." [13] The activities and events to be audited by the system are specified in control AU-2 [13], which allows each agency to set specific activities and events, and typically includes: user login attempts (both successful and unsuccessful), user logout, program start and end, attempts for privileged access (both successful and unsuccessful), and system transactions (e.g., URL requests to a web server).

Controls AU-2 and AU-3 taken together provide a basis for forensic investigations of any suspected security incidents. Security audit logging can also be useful for monitoring system activity, or for workload management and for debugging.

RMF officials expect IT systems to perform security auditing as part of their basic configuration, for the operating system and application software. AM vendors will likely have to implement at least some controls in the AU family for the IT components of their systems. If, for example, users log into the printer control software but not the operating system, the AM software would have to save audit logs of user activity to comply with RMF controls.

AM vendors should also consider the possibility that DoD agencies might also be concerned about the security and integrity of printed objects, and may require auditing for all phases of the AM production process (design/scan, simulation, build plan, printer maintenance and calibration, object printing, object testing, and object maintenance), as well as auditing access to, and inventory of, print materials, and related supply chains.

Evidence to demonstrate a system's compliance with this control can include samples of the audit logs, or system configuration for auditing (e.g., activity $\log$ and error log configurations for web server software).

\subsubsection{Security Control CA-3 "System Interconnections"} Our next example of an RMF security control is the third control in the Security Assessment and Authorization family, designated
CA-3 and titled "System Interconnections" [13]. This is a control that is frequently selected to apply to IT systems which have data connections to other systems outside of the client agency, or in other divisions of the client agency. The CA-3 control may be relevant to AM systems that connect to a network or other IT system(s). The DoD IG noted in the audit report that AM systems often had data interconnections [1], and that prior description as stand-alone systems were incorrect [1].

The control text for CA-3 is in Figure 7: [13]

The organization:

a. Authorizes connections from the information system to other information systems through the use of Interconnection Security Agreements;

b. Documents, for each interconnection, the interface characteristics, security requirements, and the nature of the information communicated; and

c. Reviews and updates Interconnection Security Agreements [Assignment: organization-defined frequency].

Figure 7: Text of RMF control CA-3 [13]

Control text in square brackets [] represent places for each agency to enter values or parameters in accordance with their own specific requirements, policies, and procedures. For CA-3, each agency defines the how often the Interconnection Security Agreements are to be reviewed and updated under Part $\mathrm{C}$ of control CA-3. Typically, annual updates are required. Note that documenting all system interconnections provides a basis for determining allowed versus unallowed network traffic, from traffic monitoring.

Control CA-3 supports system security by requiring data connections between systems to be documented, reviewed, and approved in an Interconnection Security Agreement (ISA). Data connections for AM systems would include file inputs (e.g., stl or G-code files), data from devices that monitor the AM system (e.g., temperature, sound, video), results of certification tests of the printed object(s), and maintenance records. It is incumbent upon the AM vendor to check with each agency for their specific requirements around control CA-3.

Evidence to demonstrate a system's compliance with this control would be the official, signed ISA (or acceptable alternative document).

4.4.5 Security Control CM-8 "Information System Component Inventory"

Our next example of an RMF security control is the eighth control in the Configuration Management family, designated CM 8 and titled "Information System Component Inventory" [13]. Note the DoD IG's audit report included concerns about AM systems "accounting for AM assets". [1] 
The control text for CM-8 is in Figure 8: [13]

$$
\begin{aligned}
& \text { The organization: } \\
& \text { a. Develops and documents an inventory of } \\
& \text { information system components that: } \\
& \text { 1. Accurately reflects the current information } \\
& \text { system; } \\
& \text { 2. Includes all components within the } \\
& \text { authorization boundary of the information } \\
& \text { system; } \\
& \text { 3. Is at the level of granularity deemed necessary } \\
& \text { for tracking and reporting; and } \\
& \text { 4. Includes [Assignment: organization-defined } \\
& \text { information deemed necessary to achieve } \\
& \text { effective information system component } \\
& \text { accountability]; and } \\
& \text { Reviews and updates the information system } \\
& \text { component inventory [Assignment: organization- } \\
& \text { defined frequency]. }
\end{aligned}
$$

\section{Figure 8: Text of RMF control CM-8 [13]}

Control CM-8 requires a system inventory, which includes both hardware components and major software components. It is common for agencies to have forms for recording a system's hardware and software inventory. Some agencies have a centralized inventory system. USG agencies having official documentation of, and approval for, the IT hardware and software on their networks is a key function of the RMF process [15].

For an AM system, the hardware inventory for this control can be the 3D printer, and the printer's control computer (counted as a separate component, if applicable). The software inventory would include the operating system for each device, and other major software components (e.g., print drivers, slicing software, user interfaces). Maintaining system inventory was one of the concerns in the DoD IG audit report [1].

Evidence to demonstrate a system's compliance with this control would be the system inventory list, or the system's entries in the agency's centralized inventory system (if any).

4.4.6 Security Control PE-5 "Access Control for Output Devices"

Our last example of an RMF security control is the fifth control in the Physical and Environmental Protection family, designated PE-5 and titled "Access Control for Output Devices" [13].

The control text for PE-5 is in Figure 9: [13]

The organization controls physical access to information system output devices to prevent unauthorized individuals from obtaining the output.

Figure 9: Text of RMF control PE-5 [13]
AM systems are output devices, and may be subject to this control. NIST SP 800-53 marks control PE-5 as only applying to systems categorized as Moderate or High security impact, and not to systems categorized as Low impact. However, each individual DoD agency has the authority under RMF to also require control $\mathrm{PE}-5$ for systems that are categorized as Low impact for Confidentiality, Integrity, or Availability.

The Supplemental Guidance for this control states "Controlling physical access to output devices includes, for example, placing output devices in locked rooms or other secured areas and allowing access to authorized individuals only, and placing output devices in locations that can be monitored by organizational personnel. Monitors, printers, copiers, scanners, facsimile machines, and audio devices are examples of information system output devices. Related controls: PE-2, PE-3, PE-4, PE-18." [13]

If control PE-5 is determined to be in scope for an AM system, it applies regardless of the system's location. If an AM system is in a DoD data center or office, the additional physical access controls required by PE- 5 would put additional constraints on the space the AM printer can be installed, and may limit access for vendor and maintenance personnel. If an AM system for DoD is in a private data center or a vendor's offices, and control PE-5 applies, that location must only allow access by properly authorized individuals.

Evidence to demonstrate a system's compliance with this control can be building plans, photographs of the system's location including the access controls (e.g., door locks, signage), or a site visit by the agency's RMF assessors which includes an inspection of AM system's location.

\subsection{Privacy Controls in the RMF}

SP 800-53 Revision 4 includes controls for data privacy as well as cybersecurity [13]. The RMF Privacy controls are primarily, but not exclusively, focused on protecting Personally Identifiable Information (PII), for example the PII of registered users of AM systems. Adding privacy controls was one of the significant changes in SP 800-53 from Revision 3 to Revision 4 [13].

The RMF Privacy Controls are in a separate section of SP 800-53 Revision 4 from the Security controls. The Privacy Control section includes statements that [13]:

1. The RMF Privacy controls can apply regardless of medium of PII - including paper records.

2. Definitions of PII may vary based on additional regulatory requirements for an organization.

3. Privacy concerns for an organization may extend beyond PII and its management.

4. Privacy controls may apply to a system regardless of its security categorization.

5. Privacy controls may be selected to apply to a system based on the privacy regulations, requirements, policies, guidelines, and practices that apply to the organization issuing the ATO certification.

Privacy concerns can apply to AM systems. For example, an additively manufactured custom medical device with patient identifiers may be considered as including both PII and Protected 
Health Information (PHI), as would records retained in the AM system for that object. PHI is generally treated as a more serious category of privacy data than PII. Privacy policies vary between USG agencies, even within DoD. For example, some agencies have strict privacy requirements for Social Security Numbers, and less strict ones for postal addresses. If, for example, an agency's policy is that user registration records on a computer are privacy data, then most systems for that agency will be subject to privacy requirements, including the computers associated with $\mathrm{AM}$ systems.

It is incumbent on $\mathrm{AM}$ vendors to determine the privacy requirements for their system(s), when deployed on behalf of the specific USG customer's agency and ensure compliance with those privacy requirements.

RMF Privacy controls are designed to be technology agnostic and policy neutral, similar to the RMF Security controls. RMF Privacy controls are a much smaller set of controls [13] (a total of 36 Privacy controls and control enhancements in 8 families, versus approximately 1,000 Security controls and enhancements in 18 families).

An IT or AM system can fully comply with RMF Privacy controls, but not fully meet the requirements of other privacy regulations or laws, e.g., The Privacy Act of 1974 [29], HIPAA [30] and the HIPAA Privacy Rule, or US state laws.

\subsection{Upcoming Requirements: SP 800-53 Rev. 5}

As discussed at the start of Section 4, SP 800-53 Revision 4 is the version of the RMF cybersecurity control catalog currently in use within DoD, and most of the USG. Revision 4 was officially released in April 2013, and last updated in January 2015. Revision 5 of SP 800-53 was officially released in final form in September 2020 [31], but some of the additional supporting documents for SP 80053 have yet to be officially released in final form, which necessitate delays in the adoption of Revision 5. DoD and civilian USG agencies will need time to adopt policies, procedures, and supporting systems to the new control catalog for assessments of new IT and AM systems. Then each agency can begin scheduling the re-assessments of existing ATO certifications for all their IT and AM systems with the Revision 5 control catalog.

If the RMF Control catalog revision is not specified in the contract for the system, AM vendors should confirm with their USG counterparts, as early as possible, which Revision of SP 800-53 will be used for their RMF assessment and ATO certification. Even though the update process described in the previous paragraph could take significant time, it is possible that a specific system may be in an agency's pilot program for Revision 5. IT systems with a small number of distinct components that are relatively isolated from the network, which describe many AM systems, would be good candidates for such a pilot program, as opposed to systems with more complex security postures.

Of particular interest to the AM and manufacturing community, Revision 5 adds a new control family for Supply Chain Risk Management (SCRM) and integrates SCRM aspects throughout the catalog. The new RMF focus on SCRM in SP 800-53 Revision 5 may bring additional security attention to AM systems, given their increasing importance to the DoD supply chains and readiness
[12]. AM systems are themselves vulnerable to supply chain disruptions, e.g., in print materials and repair parts. Conversely, AM experts' supply chain experience can support DoD and USG security personnel in improving their understanding of the supply chain side of SCRM issues.

SCRM and initially recommended controls are described in NIST SP 800-161 [32], titled "Supply Chain Risk Management Practices for Federal Information Systems and Organizations", prior to the publication of SP 800-53 Revision 5. SP 800-161 is being updated, and NIST expects to release a second public draft of Revision 1 in late 2021, with a final version expected in 2022 [33]. The public comment periods for SP 800-161 Revision 1 are opportunities for the AM community to provide guidance to NIST in the development and update of SCRM requirements.

NIST SP 800-161 describes the RMF process in a single document, including the examples of the SCRM controls defined in the same document. The full RMF is defined in several separate documents. SP 800-161 may be an effective RMF starting point for individuals more familiar with supply chain concerns than with cybersecurity.

\section{RMF Resources}

There are several categories of resources to learn about the RMF in more detail than this paper can provide.

\subsection{DoD Resources}

DoD has a number of resources available, many through the Defense Information Systems Agency (DISA) web site, and others via the DoD Cyber Exchange [34] site. Many of these resources are available to the general public, some are only available to those with DoD access. AM vendors working under a DoD contract may be able to obtain some materials via their ISSO. DoD resources include:

5.1.1 Security Technical Implementation Guides and Security Requirements Guides

DISA has developed Security Technical Implementation Guides (STIGs) and Security Requirements Guides (SRGs) [35] which are documents describing secure configuration standards for a variety of operating systems, software, hardware, and also for general items such as databases, or for secure application development. DISA also publishes checklists for reporting compliance with the STIGs. Some STIGs have automated scripts available to check compliance, and DISA provides software to analyze the results of these automated scans. The publicly available versions of these can be used by vendors, even if they are not vendors to DoD.

\subsection{2 eMASS}

DoD's Enterprise Mission Assurance Support Service (eMASS) is a web-based system DoD uses to automate and manage the RMF process. eMASS supports all steps of the RMF. For example, once RMF controls have been selected for a system (Step 2), the RMF compliance information can be entered into eMASS (Step 3), and assessment information and supporting evidence can also be uploaded into it (Step 4). DoD contractors are sometimes granted access to the eMASS container for their systems. Otherwise, the designated ISSO access eMASS, enters data, and provides reports 
to the contractor. For an $\mathrm{AM}$ system vendor to $\mathrm{DoD}$, or any IT vendor, awareness of eMASS and understanding the reports it generates can make the RMF process more efficient.

\subsubsection{DoD RMF Technical Advisory Group}

The DoD has an RMF Technical Advisory Group (TAG). The DoD RMF TAG publishes a guide of recommended evidence for the RMF controls. This document is For Official Use Only, and not accessible by the general public. Some DoD agencies have included the evidence guide in their instance of eMASS, and therefore the recommended evidence in included in eMASS reports of a system's RMF control status. The RMF TAG evidence guide is another document that DoD vendors may obtain from the ISSO.

\subsection{Other Security and Privacy Frameworks}

Experience with other security or privacy frameworks can support understanding of the RMF. The ISO/IEC 27001 framework [36] is an international standard for managing information security, and is similar to RMF in terms of security topics and the size of the control catalog. However, ISO/IEC 27001 [36] compliance is audited at the organizational level, whereas the RMF is assessed for each system. SP 800-53 [13] includes a mapping between RMF and ISO 27001 controls in Appendix $\mathrm{H}$ of Revision 4, and in a stand-alone document for Revision 5. Vendors familiar with ISO/IEC 27001 can gain RMF knowledge via this mapping.

The NIST Framework for Improving Critical Infrastructure Cybersecurity (called the "Cyber Security Framework" or CSF) [37] was developed by NIST "To strengthen the resilience of [critical] infrastructure" against cyber threats. The CSF document [37] includes mapping from its sub-categories to the RMF controls, and to several other cybersecurity frameworks. The CSF mapping can be used to connect different cybersecurity frameworks to the RMF controls, supporting AM vendors' improving their RMF understanding based on experience with other cybersecurity frameworks. NIST SP 800-37 [15] (which describes the overall RMF process) includes the NIST CSF as a reference for several of the RMF tasks listed in SP 800-37, and also states that CSF Profiles "can provide a link between cybersecurity activities and organizational mission/business objectives, which supports riskbased decision-making throughout the RMF." [15]

$\mathrm{DoD}$ is beginning to require its vendors that handle Controlled Unclassified Information (CUI) or Federal Contract Information (FCI) be assessed for their organizational cybersecurity maturity, under the Cybersecurity Maturity Model Certification (CMMC) program [38]. CMMC is designed to address that "Malicious cyber actors have targeted, and continue to target the Defense Industrial Base (DIB) sector and the supply chain of the Department of Defense (DoD)" [38]. AM systems that are part of the DoD supply chain may be subject to CMMC certification requirements once CMMC has been fully implemented, and possibly as part of CMMC pilot programs over the next few years. Experience with the cybersecurity topics in RMF can support compliance with the newer CMMC program, and learning about the CMMC program can support improved understanding of RMF. It is notable that of the 17 security domains within CMMC, 15 have the same titles as
RMF control families, and one additional CMMC domain is a topic that is also addressed by specific RMF controls.

\section{Conclusions}

DoD has in recent years taken advantage of AM's promise of democratizing and distributing manufacturing, reducing waste, and enabling rapid production of new and specialty products, in support of DoD's mission [12]. As AM and AM parts become an increasingly important to DoD's supply chain, the cyber threat landscape within the AM world becomes more visible, relevant, and impactful. The DoD IG audit of AM cybersecurity [1] identified significant gaps in AM systems' compliance with existing DoD cybersecurity regulations. The IG's recommendations from the audit attempt to address those gaps, by requiring AM system to follow the NIST RMF, and to comply with appliable security and privacy controls to obtain ATO certification. The DoD CIO, in response to the IG audit, confirmed that RMF compliance and ATO certification was already required by DoD [1].

This paper reviewed the IG audit [1], described the NIST RMF process [15], highlighted example controls from the security and privacy catalogs, and listed resources for AM system vendors to understand and comply with the NIST RMF.

It is our hope that the AM community will leverage cybersecurity frameworks such as the NIST RMF, and minimize the impact to the AM world of the possible cybersecurity issues from the IT world.

\section{DISCLAIMER}

This communication contains general information only, and none of Deloitte Touche Tohmatsu Limited, its member firms, or their related entities (collectively, the "Deloitte Network") is, by means of this communication, rendering professional advice or services. Before making any decision or taking any action that may affect your finances or your business, you should consult a qualified professional adviser. No entity in the Deloitte Network shall be responsible for any loss whatsoever sustained by any person who relies on this communication.

\section{ABOUT DELOITTE}

Deloitte refers to one or more of Deloitte Touche Tohmatsu Limited, a UK private company limited by guarantee ("DTTL"), its network of member firms, and their related entities. DTTL and each of its member firms are legally separate and independent entities. DTTL (also referred to as "Deloitte Global") does not provide services to clients. In the United States, Deloitte refers to one or more of the US member firms of DTTL, their related entities that operate using the "Deloitte" name in the United States and their respective affiliates. Certain services may not be available to attest clients under the rules and regulations of public accounting. Please see www.deloitte.com/about to learn more about our global network of member firms. 


\section{ACKNOWLEDGMENTS}

The authors would like to thank Jacqueline Gainer, Roger D. Hill, Kelly Marchese, Mark Nace, and Shunda Threat for helpful reviews of this manuscript. The authors would also like to thank the two anonymous reviewers for their critical reading, and for suggestions that helped improve and clarify this manuscript.

\section{REFERENCES}

[1] Department of Defense Office of Inspector General. 2021. Audit of the Cybersecurity of Department of Defense Additive Manufacturing Systems (DODIG-2021-098). https://www.dodig.mil/reports.html/article/2683843/audit-ofthe-cybersecurity-of-department-of-defense-additive-manufacturing-syst/ Full report at: https://media.defense.gov/2021/Jul/07/2002757308/-1/-1/1/DODIG2021-098.PDF

[2] NIST Information Technology Laboratory Computer Security Resource Center 2021. About the Risk Management Framework (RMF): A Comprehensive, Flexible, Risk-Based Approach https://csrc.nist.gov/projects/riskmanagement/about-rmf

[3] Federal Office for Information Security. 2014. The state of IT security in Germany 2014

https://www.bsi.bund.de/SharedDocs/Downloads/EN/BSI/Publications/Securitysi tuation/IT-Security-Situation-in-Germany-2014.pdf?_blob=publicationFile\&v=1 ,p. 31

[4] Sofia Belikovetsky, Yosef A. Solewicz, Mark Yampolskiy, Jinghui Toh, and Yuval Elovici. 2019. Digital Audio Signature for 3D Printing Integrity. In IEEE Transactions on Information Forensics And Security, 14 (5), 1127-1141

[5] NIST Information Technology Laboratory National Vulnerability Database. 2021. https://nvd.nist.gov/

[6] Zhanna Smith, Eugenia Lostri, James Lewis. 2020. The Hidden Costs of Cybercrime. https://www.csis.org/analysis/hidden-costs-cybercrime

[7] Jack Evans. 2021. "Someone tried to poison Oldsmar's water supply during hack, sheriff says”. https://www.tampabay.com/news/pinellas/2021/02/08/someonetried-to-poison-oldsmars-water-supply-during-hack-sheriff-says/

[8] Natasha Bertrand, Evan Perez, Zachary Cohen, Geneva Sands and Josh Campbell. 2021. "Colonial Pipeline did pay ransom to hackers, sources now say". https://edition.cnn.com/2021/05/12/politics/colonial-pipeline-ransomwarepayment/index.html

[9] Simon Goldenberg, John Brown, Jeff Haid, and John Ezzard. 2016. 3D opportunity and cyber risk management: Additive manufacturing secures the thread. https://www2.deloitte.com/us/en/insights/focus/3d-opportunity/3dprinting-cyber-risk-management.html

[10] Mark J. Cotteleer, Stuart Tronton, Ed Dobner. 2016. 3D opportunity and the digital thread: Additive manufacturing ties it all together. https://www2.deloitte.com/content/dam/insights/us/articles/3d-printing-digitalthread-in-manufacturing/ER_3060-3D-opp-_Digital-Thread_MASTER-1.pdf

[11] Deb Golden, Kelly Marchese. 2017. The Additive Cyber Risk of Additive Manufacturing: Six Steps Towards Greater Security in the Supply Chain. Webinar for Industry Week magazine

[12] Joint Defense Manufacturing Council. 2021. Department of Defense Additive Manufacturing Strategy. https://www.cto.mil/wp-content/uploads/2021/01/dodadditive-manufacturing-strategy.pdf

[13] Joint Task Force Transformation Initiative. 2013. NIST Special Publication 800-53 Revision 4: Security and Privacy Controls for Federal Information Systems and Organizations https://csrc.nist.gov/publications/detail/sp/800-53/rev-4/final

[14] Keith Stouffer, Timothy Zimmerman, CheeYee Tang, Joshua Lubell, Jeffrey Cichonski, Michael Pease, John McCarthy. 2020. NIST Internal Report NISTIR 8183 Revision 1: Cybersecurity Framework Version 1.1 Manufacturing Profile. https://nvlpubs.nist.gov/nistpubs/ir/2020/NIST.IR.8183r1.pdf

[15] Joint Task Force. 2018. NIST Special Publication 800-37 Revision 2: Risk Management Framework for Information Systems and Organizations: A System Life Cycle Approach for Security and Privacy. https://csrc.nist.gov/publications/detail/sp/800-37/rev-2/final
[16] Federal Information Security Modernization Act of 2014, Pub. L. 113-283

[17] NIST Information Technology Laboratory Computer Security Resource Center. 2006. FIPS 200: Minimum Security Requirements for Federal Information and Information Systems. https://csrc.nist.gov/publications/detail/fips/200/final

[18] Committee on National Security Systems. 2014. CNNS Instruction (CNSSI) No 1253 Security Categorization and Control Selection for National Security Systems. https://www.cnss.gov/cnss/issuances/Instructions.cfm and search for 1253

[19] Department of Defense. 2019. DoD Instruction 8500.01: Cybersecurity. https://www.esd.whs.mil/Portals/54/Documents/DD/issuances/dodi/850001_2014 .pdf

[20] Department of Defense. 2020. DoD Instruction 8510.01: Risk Management Framework (RMF) for DoD Information Technology (IT) https://www.esd.whs.mil/Portals/54/Documents/DD/issuances/dodi/851001p.pdf? ver=qEE2HGN_HE4Blu7161t1TQ\%3d\%3d

[21] Committee on National Security Systems. 2015. CNSS Instruction (CNSSI) No 4009: National Information Assurance Glossary. https://www.cnss.gov/cnss/issuances/Instructions.cfm and search for 4009

[22] 44 USC $\S 3542 f$

[23] Deloitte. 2020. RMF Overview and Roles \& Responsibilities. Internal presentation

[24] NIST Computer Security Division, Information Technology Laboratory. 2004 Federal Information Processing Standards Publication (FIPS PUB) 199: Standards for Security Categorization of Federal Information and Information Systems. https://nvlpubs.nist.gov/nistpubs/fips/nist.fips.199.pdf

[25] Kevin Stine, Rich Kissel, William C. Barker, Jim Fahlsing, and Jessica Gulick. 2008. NIST SP 800-60 Vol. I (Rev. 1): Guide for Mapping Types of Information and Information Systems to Security Categories https://nvlpubs.nist.gov/nistpubs/Legacy/SP/nistspecialpublication800-60v1r1.pdf

[26] Kelley Dempsey, Nirali Shah Chawla, Arnold Johnson, Ronald Johnston, Alici Clay Jones, Angela Orebaugh, Matthew Scholl, Kevin Stine. 2011. NIST Special Publication 800-137: Information Security Continuous Monitoring (ISCM) for Federal Information Systems and Organizations. https://nvlpubs.nist.gov/nistpubs/Legacy/SP/nistspecialpublication800-137.pdf

[27] FedRAMP. 2021. Security Cloud Services for the Federal Government https://www.fedramp.gov/

[28] FedRAMP. 2021. Documents and Templates. https://www.fedramp.gov/documents-templates/

[29] The Privacy Act of 1974, as amended, 5 USC $§ 552 a$

[30] Health Insurance Portability and Accountability Act, Pub. L. No. 104-191

[31] NIST Information Technology Laboratory Computer Security Resource Center 2020. SP 800-53 Rev. 5: Security and Privacy Controls for Information Systems and Organizations. https://csrc.nist.gov/News/2020/sp-800-53-revision-5published

[32] Jon Boyens, Celia Paulsen, Rama Moorthy, Nadya Bartol. 2015. SP 800-161: Supply Chain Risk Management Practices for Federal Information Systems and Organizations. https://csrc.nist.gov/publications/detail/sp/800-161/final

[33] NIST Information Technology Laboratory Computer Security Resource Center. 2021. SP 800-161 Rev. 1 (Draft): Cyber Supply Chain Risk Management Practices for Systems and Organizations. https://csrc.nist.gov/publications/detail/sp/800161/rev-1/draft

[34] DISA. 2021. DoD Cyber Exchange public web site https://public.cyber.mil/

[35] DISA. 2021. Security Technical Implementation Guides (STIGs) https://public.cyber.mil/stigs/

[36] ISO. 2021. Popular Standards: ISO/IEC 27001 Information Security Management https://www.iso.org/isoiec-27001-information-security.html

[37] NIST. 2021. Framework for Improving Critical Infrastructure Cybersecurity (Cybersecurity Framework). https://www.nist.gov/cyberframework

[38] Office of the Under Secretary of Defense for Acquisition \& Sustainment. 2021 Cybersecurity Maturity Model Certification. https://www.acq.osd.mil/cmmc/ 